
\title{
25 Research Soure \\ Dynamic alteration in the gut microbiota and metabolome during the pregnancy
}

\section{Peifeng Xie}

Institute of Subtropical Agriculture Chinese Academy of Sciences

Chengjun Hu

Institute of Subtropical Agriculture Chinese Academy of Sciences

Qinghua He

Shenzhen University

Qian Zhu

Institute of Subtropical Agriculture Chinese Academy of Sciences

Xiangfeng Kong ( $\square$ nnkxf@isa.ac.cn )

Institute of Subtropical Agriculture Chinese Academy of Sciences https://orcid.org/0000-0001-64066445

\section{Research Article}

Keywords: gut microbiota, Huanjiang mini-pigs, metabolites, obesity, pregnancy

Posted Date: March 18th, 2021

DOI: https://doi.org/10.21203/rs.3.rs-319234/v1

License: (c) (1) This work is licensed under a Creative Commons Attribution 4.0 International License.

Read Full License 


\section{Abstract \\ Background}

Gut microbiota and their metabolites were associated with obesity. Our previous study showed that maternal body fat percentage increased from days 45 to 110 of gestation in a Huanjiang mini-pig model. Thus, 16S rRNA sequencing and metabonomic techniques were used to investigate the changes of maternal gut microbiota composition and microbial metabolite profile from days 45 to 110 of gestation.

\section{Results}

The abundances of Clostridium_sensu_stricto_1, Romboutsia, Turicibacter, and Streptococcus in jejunum contents were higher in day 110 than those in day 45 or 75 of gestation. In ileum, the abundance of Streptococcus was the highest $(P<0.05)$ at day 110 of gestation, as well as the metabolism function of jejunal and ileal microbiota. The ileal butyrate and acetate concentrations were the highest at day 45 and day 110 of gestation, respectively. In colon, the concentrations of cadaverine and spermine were the highest $(P<0.05)$ at days 45 and 110 of gestation, respectively. Metabonomic analysis demonstrated that metabolic pathways including glutamine and glutamate metabolism, phenylalanine, tyrosine and tryptophan biosynthesis, and alanine, aspartate, and glutamate metabolism changed during gestation.

\section{Conclusions}

Microbiota composition and metabolites changed dramatically from the early to the late pregnancy, which might be associated with the maternal fat accumulation.

\section{Background}

To support the growth and development of conceptus, a multitude of physiological and biochemical changes were observed during pregnancy, which were partly associated with long-term health problems in the offspring [1]. Gut microbiota impacts body physiology and is associated with the etiology of various diseases, including obesity, type 2 diabetes [2], and insulin resistance [3]. Evidence showed that maternal metabolism was associated with the changes of their gut microbiota composition [4]. In addition, the pregnancy metabolic symptom is associated with changes in the gut microbiota composition in third trimester of pregnancy [5]. These findings indicate that gut microbiota play an important role in maternal metabolism during gestation.

Intestinal microbiota shapes host physiology through their metabolites. Intestine microbiota metabolites, such as short-chain fatty acids (SCFAs) and bioamines, play important roles in host physiology [6]. The SCFAs are mainly produced by colonic microbiota from dietary carbohydrates and proteins [7], and are associated with obesity and metabolic syndromes [8]. For instance, butyrate protects against diet-induced 
obesity by increasing energy expenditure [9], and acetate mediates a microbiome-brain- $\beta$-cell axis to promote metabolic syndrome [10]. Therefore, to evaluate the influence of the microbiota composition on host metabolism, it is important to measure the microbiota metabolites, which will help us figure out the relationship between gut microbiota, metabolites, and host.

Intestine microbiota and their metabolites play important role in obesity. We previously observed maternal obesity at the late pregnancy in a sow model [11]. However, the changes of intestine microbiota and their metabolites during gestation extend are still unknown. Mini-pig have served as one of the animal model in clinical medicine application on human pregnancy [12]. Therefore, we used 16S rRNA sequencing and nuclear magnetic resonance (NMR) based-metabolomics to investigate the gut microbiota composition and identify metabolite markers from days 45 to 110 of pregnancy in a Mini-pig model.

\section{Materials And Methods}

\section{Animals, housing, and treatment}

A total of 24 primiparous Huanjiang mini-pigs with an average initial body weight (BW) of $30 \mathrm{~kg}$ were obtained from a farm located in Huanjiang County, Guangxi Province, China. The sows were randomly assigned to one of eight pens, with three sows per pen. The animals were fed a diet formulated according to the recommendations of the Chinese National Feeding Standard for Swine (Supplemental Table 1). All sows were housed in $2 \mathrm{~m} \times 3 \mathrm{~m}$ pens with cement-sclerified flooring. Each pen was equipped with a feeder and a nipple drinker. All sows had ad libitum access to drinking water and were fed three times daily (about $2 \%$ of BW) [13].

\section{Sample collection and preparation}

At day 45 (early-pregnancy), 75 (Mid-pregnancy), and 110 (late-pregnancy) of pregnancy, the sows were sacrificed using electrical stunning and exsanguination. The middle jejunal content was collected, and the ileal and colonic luminal contents from a region $10 \mathrm{~cm}$ anterior and posterior to the ileocecal valve were collected, respectively $[14,15]$. All samples were collected into sterile tubes and stored at $-80^{\circ} \mathrm{C}$ for further analysis.

\section{DNA extraction and PCR amplification}

Total genomic DNA was extracted from the intestinal content samples using HiPure Stool DNA Kits (Magen, Guangzhou, China) according to the manufacturer's instructions. The concentrations of DNA were measured using a NanoDrop ND-1000 spectrophotometer (NanoDrop Technologies Inc., Wilmington, $D E$, USA). Total DNA of the intestinal contents were diluted to $50 \mathrm{ng} / \mu \mathrm{L}$ and then used in the preparation of amplicons for high-throughput sequencing.

The V3-V4 hypervariable regions of the bacteria 16S rRNA gene were amplified as described previously [14]. The PCR reaction volumes $(10 \mu \mathrm{L})$ comprised $4 \mu \mathrm{L}$ of $5 \times$ FastPfu Buffer, $2 \mu \mathrm{L}$ of $2.5 \mathrm{mM}$ dNTPs, 0.8 $\mu \mathrm{L}$ of each primer $(5 \mu \mathrm{M}), 0.4 \mu \mathrm{L}$ of FastPfu Polymerase, and $2 \mu \mathrm{L}$ DNA. The PCR reactions were 
conducted using the following program: 3 min of denaturation at $95^{\circ} \mathrm{C} ; 27$ cycles of $30 \mathrm{~s}$ at $95^{\circ} \mathrm{C}, 30 \mathrm{~s}$ for annealing at $55^{\circ} \mathrm{C}$, and $45 \mathrm{~s}$ for elongation at $72^{\circ} \mathrm{C}$; and a final extension at $72^{\circ} \mathrm{C}$ for $10 \mathrm{~min}$. The PCR products were extracted from a $2 \%$ agarose gel and further purified using the AxyPrep DNA Gel Extraction Kit (Axygen Biosciences, Union City, CA, USA) and quantified using QuantiFluor ${ }^{\mathrm{T}}$-ST (Promega, USA) according to the manufacturer's protocol. Purified amplicons were operated using paired-end sequencing by Illumina MiSeq (Illumina, San Diego, USA). The instructions of the platform and the manufacturer were from a commercial service provider (Majorbio, Shanghai, China).

\section{Processing of sequencing data}

Raw fastq files were demultiplexed, quality-filtered by Trimmomatic (v.0.30), and merged by FLASH (v.1.2.11) with the following criteria: (i) The reads were truncated at any site receiving an average quality score $<20$ over a 50 bp sliding window; (ii) Primers were exactly matched allowing 2 nucleotides mismatching and reads containing ambiguous bases were removed; (iii) Sequences with overlap $>10 \mathrm{bp}$ were merged according to their overlap sequence. Operational taxonomic units (OTUs) were clustered with $97 \%$ similarity cutoff using UPARSE (v7.1 http://drive5.com/ uparse/) and chimeric sequences were identified and removed using UCHIME. The taxonomy of each 16S rRNA gene sequence was analyzed by Ribosomal Database Project Classifier algorithm (http://rdp.cme.msu.edu/) against the Silva (SSU123) 16S rRNA database using confidence threshold of 70\% [16]. The ACE, Cha01, and Simpson for microbiota were used to estimate alpha diversity values. The unadjusted means of OTU-level microbial abundances was analyzed using Partial least squares discriminant analysis (PLS-DA).

\section{Intestine metabolites analysis in intestine contents}

The SCFAs including acetate, propionate, butyrate, iso-butyrate, valerate, and isovalerate were analyzed using gas chromatography as described previously [13]. Bioamines including putrescine, cadaverine, spermidine, spermine, and tyramine were measured using high-performance liquid chromatography as described previously [13].

\section{${ }^{1} \mathrm{H}$ NMR spectroscopy analysis}

${ }^{1} \mathrm{H}$ NMR spectra were conducted according to a previous study [17] . In briefly, the $90^{\circ}$ pulse length ( 10.0 $\mathrm{ms}$ ) was adjusted individually for each sample. The transients were collected into $32 \mathrm{k}$ data points for sample, with a spectral width of $20 \mathrm{ppm}$ and a recycle delay of $2.0 \mathrm{~s}$. The NMR spectral data scaled to unit variance were analyzed by the orthogonal projection to latent structure with discriminant analysis (OPLS-DA) method [17]. Principal component analysis (PCA) was performed to identify metabolic differences among the groups. Each metabolite was assigned a variable importance in the projection value according to the PLS-DA results. Metabolites with fold change variable importance in the variable importance in the projection (VIP) value $>1$ and $P$ value $<0.05$ were used to select the significant metabolites between the three gestation stages.

\section{Statistical analysis}


The alpha diversity indices of microbiota communities and colonic metabolites were analyzed using Oneway analysis of variance and Duncan's multiple-range post hoc test in SAS (SAS Institute, Inc., Cary, NC). Student's t-tests were employed to compare two groups. The relative abundances at phyla and genera levels of microbial communities were analyzed using the Kruskal Wallis test. The correlation between microbiota and metabolic parameters was analyzed using Pearson's linear correlation coefficient. Differences with $P$ value $<0.05$ were considered as statistically significant, whereas a tendency was considered to exist at $0.05 \leq P<0.10$.

\section{Results}

\section{Diversity of intestine microbiota communities}

After size filtering, quality control, and chimera removal, 1,997,373 high-quality reads were obtained. The average read length was $440 \mathrm{bp}$. All the samples were normalized and the OTU table within each sample was rarefied to 22,701 sequence reads based on the sample rarefaction curves and Shannon curves (Supplemental Figure 1). A Venn diagram was used to investigate the core microbiota presented in intestinal contents with different pregnancy stages (Supplemental Figure 2). There were 555, 321 and 984 OTUs shared in jejunum, ileum, and colon contents, respectively.

The normalized sequence reads were used to calculate richness and diversity indices. As gestation extend, jejunal and ileal contents were showed increase $(P<0.05)$ in ACE and Chao1 indices (Figure 1AF). In colonic contents, Simpson index trended to decrease $(P=0.06)$ along with pregnancy (Figure $2 \mathrm{G}-\mathrm{I})$.

PLS-DA was used to analyze the differences among the three groups (Figure 1J); samples from the jejunum and ileum were clustered together. For colonic contents, the samples collected at days 45,75 , and 110 of gestation were separated with each other.

\section{Composition of intestinal microbiota communities}

At phylum level, the dominant microbes in jejunum and ileum were Firmicutes, and that in colon were Firmicutes and Bacteroidetes (Figure 2A). The abundances of Firmicutes and Actinobacteria were lower, whereas that of Bacteroidetes and Spirochete were higher in colon than in jejunum or ileum. In ileum, the abundance of Tenericutes increased $(P<0.05)$, and that of Firmicutes had a trend to decrease $(P=0.06)$ from days 45 to 110 of gestation (Figure $2 C$ ).

At genus level, the dominant genera in jejunum and ileum were Lactobacillus and Clostridium, and that in colon were Bacteroidales S24-7 group, Lachnospiraceae XPB1014 group, and Lactobacillus (Figure 3A). In jejunum, the abundances of Clostridium_sensu_stricto_1, Romboutsia, Turicibacter, and Streptococcus increased $(P<0.05)$, and the abundance of Megasphaera decreased $(P<0.05)$ from days 45 to 110 of gestation (Figure 3B). In ileum, the abundance of Streptococcus was higher $(P<0.05)$ at day 110 than that at day 45 and day 75 of gestation (Figure 3C). In colon, the abundances of [Eubacterium]_coprostanoligenes_group and Streptococcus increased $(P<0.05)$, while 
Clostridium_sensu_stricto_1 and Ruminococcaceae_UCG-014 decreased $(P<0.05)$ from days 45 to 110 of gestation (Figure 3D).

\section{Intestine metabolite profiles}

Table 1 showed the SCFAs concentrations in intestinal contents. In jejunum, the acetate concentration was lower $(P<0.05)$ at day 75 than that at day 45 of gestation. The ileal acetate concentration increased linearly while the butyrate concentration decreased $(P<0.05)$ from days 45 to 110 of gestation. The acetate concentration in colonic content decreased $(P<0.05)$ from days 45 to 110 of gestation.

Table 2 showed the bioamine concentrations in jejunal, ileal, and colonic contents. In jejunum, the putrescine concentration was lowest $(P<0.05)$, and the concentrations of spermidine and spermine were highest $(P<0.05)$ at day 110 of gestation. In ileum, the putrescine concentration was lower $(P<0.05)$ at day 110 than that at days 45 and 75 of gestation. In colon, the concentrations of cadaverine and spermine were highest $(P<0.05)$ at day 45 and day 110 of gestation, respectively.

\section{Correlation between metabolites and microbiota}

In jejunum (Figure 4A), the putrescine concentration had negative correlation with the abundances of Terrisporobacter, Clostridium_sensu_stricto_1, and Turicibacter, while the spermidine concentration had positive correlation with the abundances of Terrisporobacter, Clostridium sensu stricto 1, Turicibacter, and family Peptostreptococcaceae. The spermine concentration had negative correlation with the abundances of Megasphaera and Olsenella.

In ileum (Figure 4B), the acetate concentration had positive correlation with the abundances of Streptococcus, Corynebacterium 1, Turicibacter, family Peptostreptococcaceae, and Bifidobacterium, but had negative correlation with the abundance of Lactobacillus.

In colon (Figure 4C), the cadaverine concentration had negative correlation with the abundances of Streptococcus and [Eubacterium] coprostanoligenes_group. The putrescine concentration had negative correlation with the abundance of unclassified_f_Lachnospiraceae. The isobutyrate concentration had positive correlation with the abundances of Christensenellaceae R-7 group, Treponema_2, and Ruminococcaceae UCG-005. In addition, the isovalerate concentration had positive correlation with the abundance of Christensenellaceae R-7 group.

\section{Function prediction of microbiota communities}

The function prediction analysis for jejunal and ileum microbiota showed that eight metabolism functions, including energy production and conversion, amino acid transport and metabolism, inorganic ion transport and metabolism, carbohydrate transport and metabolism, nucleotide transport and metabolism, coenzyme transport and metabolism, lipid transport and metabolism and secondary metabolites biosynthesis, and transport and catabolism were increased $(P<0.05)$ from days 45 to 110 of gestation (Figure 5A-D). 


\section{Metabonomic analysis}

To further investigate whether the gestation stage affected the metabolite profiles in intestine contents, the ${ }^{1} \mathrm{H}$ NMR spectroscopy analysis was used to analyze the metabolite profiles (Supplemental Figure 3 ). The PCA score plots (Figure 6A) showed distinct separation in the jejunum, ileum and colon. The PLS-DA model showed that sample from the three gestation stages (days 45 VS 75, and 75 VS 110) were wellseparated (Figure 6B-G). The concentrations of 17 metabolites, including leucine, isoleucine, valine, alanine, citrulline, arginine, lysine, proline, methionine, glutamate, pyruvate, succinate, glutamine, asparagine, choline, threonine, and tyrosine in jejunum decreased $(P<0.05)$ from days 45 to 75 of gestation (Figure 7A). In addition, the concentrations of leucine, isoleucine, valine, alanine, citrulline, arginine, lysine, proline, methionine, and tryptophan decreased $(P<0.05)$ at day 45 compared to those at day 110 of gestation (Figure 7A). From days 45 to 110 of gestation, the concentrations of isoleucine, methionine, glutamate, glutamine, pyruvate, and aspartate in ileum decreased $(P<0.05)$ (Figure 7B), while the concentrations of leucine, isoleucine, arginine, proline, methionine, glutamate, pyruvate, glutamine, aspartate, asparagine, choline, glycine, creatine, histidine, and tyrosine in colon increased $(P<$ 0.05) from day 75 to day 110 of gestation (Figure 7C).

\section{Metabolic pathway analysis}

MetaboAnalyst 4.0 was employed to explore the metabolic pathways in intestine contents from early to late gestation. The identified metabolites with $P<0.05$ and VIP $>1$ (Supplemental table 2-4) were used to perform pathway analysis. A series of metabolic pathways were affected by gestation stage. In jejunum, the pathways with significant interferences were glutamine and glutamate metabolism, taurine and hypotaurine metabolism, alanine, aspartate and glutamate metabolism, pyruvate metabolism, glycolysis or gluconeogenesis (Figure 8A, B). The primary metabolic pathways impacted in ileum were glutamine and glutamate metabolism, phenylalanine, tyrosine and tryptophan biosynthesis, glycolysis or gluconeogenesis, alanine, aspartate and glutamate metabolism, arginine and proline metabolism (Figure $8 \mathrm{C}, \mathrm{D})$, and that in colon were glutamine and glutamate metabolism, phenylalanine, tyrosine and tryptophan biosynthesis, valine, leucine and isoleucine biosynthesis, alanine, aspartate and glutamate metabolism (Figure 8E, F).

\section{Discussion}

Gut microbiota and their metabolites exert important role in maternal metabolism and physiology. Therefore, this study was to investigate the gut microbiota composition and their metabolites from early (day 45 ) to the late (day 110) of gestation. It was found that the microbiota richness and diversity in jejunum and ileum increased from days 45 to 110 of gestation, and identified several microbiota and metabolites using a microbiota-metabolome analysis. Moreover, these findings also demonstrated that acetate concentration increased in ileum but decreased in colon from days 45 to 110 of gestation. It is suggested a marked influence of gestation stage on intestine microbial community and metabolic profiles, which might be involved in maternal fat accumulation at late gestation. 
In the present study, the jejunal and ileal microbiota richness and diversity increased from days 45 to 110 of gestation, as indicated by the ACE, Chao1, and Simpson indices. However, the gestation stages exert no effects on colonic microbiota richness, with no difference observed in the ACE and Chao 1 values. These results were in line with a previous study which reported that gestation stage did not alter colonic microbiota richness in gestating sows [13]. By contrast, several studies showed that the fecal microbiota diversity and richness were lower at the end than those at the beginning of pregnancy $[18,19]$. This discrepancy might be explained partially by differences in the animal model. The animal model used in the present study was sow, whereas that used in studies of Koren et al. (2012) and Kennedy et al. (2016) was human and rat, respectively. The PLS-DA showed that the microbiota in jejunal and ileal contents at days 45,75 , and 110 of pregnancy were clustered together, but were separated with the colonic samples, suggesting small intestine and colon showed different microbiota composition. In addition, the colon samples were separated with each other, indicating that microbiota composition in colon presented enormous dynamic changes over the course of pregnancy.

We also found that the dominant phyla in jejunal and ileal contents was Firmicutes, and those in colonic contents were Firmicutes, Bacteroidetes, and Actinobacteria, which is consistence with previous studies $[15,13]$. In addition, lower Firmicutes abundance and Firmicutes/Bacteroidetes ratio were observed in the ileum from days 45 to 110 of gestation, which is in line with the changes of body fat percentage of sows [11]. However, earlier studies showed that obesity were associated with increased Firmicutes abundance and Firmicutes/Bacteroidetes ratio [20, 21]. This discrepancy might be explained by differences in the physiology of animal, diets, and tested samples.

Small intestine and colon also showed a marked different microbiota type at the genus level. The dominant genera in jejunal and ileal contents were Lactobacillus and Clostridium, and that in colonic was norank_f_Bacteroidetes_S24-7_group. Studies in vitro and in vivo showed that Lactobacillus could reduce the fat storage $[22,23]$ and protect animal against obese-insulin resistance [24]. In addition, colonization of Lactobacillus in the intestine was beneficial to the health and prevented the colonization of opportunistic pathogens [25]. In the present study, the abundance of Lactobacillus reduced in jejunum and ileum ongoing with pregnancy. Consistence with the changes of Lactobacillus abundance, the sows are more obese at the late pregnancy [11], indicating that Lactobacillus was associated with changed maternal metabolism during the pregnancy. Obese women had higher number of mutants Streptococcus compared to normal-weight women [26], and higher Streptococcus colonization was also significantly associated with obesity in children [27]. Here, the highest abundance of Streptococcus in jejunum, ileum, and colon at day 110 of gestation suggested that Streptococcus might contribute to maternal obesity. However, the mechanism still needs further investigation. The increase of Clostridium sensu stricto 1 along with the pregnancy may have connections with the inflammation in the jejunum and ileum [28]. Other studies also showed that some cluster of Clostridium produced butyrate and played a role in antiinflammation [29, 30]. Based on these previous studies, we suspected that the increased abundances of Clostridium sensu stricto 1 in jejunum might contribute to the improvement of jejunum health as gestation extend. 
Our previous studies indicated that the SCFAs were associated with body fat weight [14, 31]. Therefore, we investigate whether gut SCFAs concentration changed as gestation extend. The present study showed that the main SCFAs in jejunal and ileal contents were acetate and butyrate; while those in colonic contents were acetate, propionate, and butyrate. Acetate was considered to be preferentially used for energy generation and lipogenesis in all kinds of tissues [32]. Butyrate increases energy expenditure and prevents diet-induced obesity and insulin resistance $[9,33]$. Consistence with the changes of maternal body fat percentage [11], the ileal acetate concentration increased and the butyrate concentration decreased from days 45 to 110 of gestation, indicating that acetate and butyrate in ileum might contribute to maternal obesity at late pregnancy. Based on these findings, it was summarized that acetate and butyrate might be the main SCFAs that involved in maternal fat metabolism during the pregnancy.

Gestation stage also leads to different microbial function and metabolite profiles in intestine. Microbiota in small intestine and colon showed different function, with eight functions (shown in Fig. 5) increased in jejunum and ileum, whereas these functions showed no differences in colon. The discrepancy could explain by differences in microbiota composition. The main dominant genus in jejunum and ileum were Lactobacillus and Clostridium, whereas that in colon were norank_f_Bacteroidetes_S24-7_group, Treponema_2, and Lachnospiraceae_XPB1014_group. To investigate the gut microbiota with their functional states, a metabolomics analysis was conducted in this study. PLS-DA analysis showed a clear cluster of metabolic profile in different gestation stage, indicating significant differences in metabolites profiles from days 45 to 110 of gestation. At day 75 of gestation, the concentration of most amino acids in jejunum was the lowest. Consistent with the change in jejunum, the concentrations of amino acids in ileum decreased as gestation extend. The main role of jejunum and ileum is responsible for nutrient digestion and absorption. Decreased amino acids concentration partly indicated the digestion and absorption function of maternal small intestine improved as gestation extend. These results also indicated that the most influenced metabolic pathway in intestine was glutamine and glutamate metabolism. The fetus grows quickly from day 75 to late gestation [34] and mother need to obtain adequate nutrients at this gestation stage to support the fetus growth. Therefore, it is suspected that the increased metabolism functions of microbiota at late pregnancy might help the mother acquire extra nutrients. Collectively, this study showed that gut metabolites changed dramatically from the early to the late pregnancy, which might be associated with maternal physiology.

\section{Conclusion}

In summary, the present study revealed that metabolism function of jejunal and ileal microbiota community increased from days 45 to 110 of gestation. As gestation extend, ileal acetate concentration increased and butyrate concentration decreased, which were associations with the abundance of specific microbiota genera. These findings can help us to understand the changes of gut microbiota communities and metabolites from the early to the late pregnancy, and provide new targets in formulating the nutritional intervention to ameliorate the adverse effects of maternal obesity on offspring health outcomes. 


\section{Abbreviations}

BW: body weight; NMR: nuclear magnetic resonance; OTUs: operational taxonomic units; OPLS-DA: orthogonal projection to latent structure with discriminant analysis; PCA: principal component analysis; PLS-DA: Partial least squares discriminant analysis; SCFAs: short-chain fatty acids; VIP: variable importance in the projection;

\section{Declarations}

\section{Acknowledgements}

The present study was jointly supported by the National Key Research and Development Project (2018YFD0500404-4), National Natural Science Foundation of China (31772613), and Special Funds for Construction of Innovative Provinces in Hunan Province (2019RS3022).

\section{Authors' contributions}

$\mathrm{XPF}, \mathrm{HCJ}$ and ZQ performed the animal feeding experiment and samples analysis. XPF and HCJ wrote the manuscript. KXF and HQH contributed to experimental concepts and design, provided scientific direction, and finalized the manuscript. All authors read and approved the final manuscript.

\section{Ethics approval and consent to participate}

The present study was carried out in accordance with the Chinese guidelines for animal welfare and experimental protocols and approved by the Animal Care and Use Committee of the Institute of Subtropical Agriculture, Chinese Academy of Sciences.

\section{Availability of data and materials}

The data generated or analyzed during the current study are available from the corresponding author by reasonable request.

\section{Consent for publication}

Not applicable.

\section{Competing interests}

The authors declare that no competing interests exist. The manuscript has not been published previously

\section{References}

1. Lahti-Pulkkinen M, Bhattacharya S, Wild SH, Lindsay RS, Raikkonen K, Norman JE, et al. Consequences of being overweight or obese during pregnancy on diabetes in the offspring: a record 
linkage study in Aberdeen. Scotland Diabetologia. 2019;62:1412-9.

2. Karlsson FH, Tremaroli V, Nookaew I, Bergström G, Behre CJ, Fagerberg B, et al. Gut metagenome in European women with normal, impaired and diabetic glucose control. Nature. 2013;498:99.

3. Ussar S, Griffin NW, Bezy O, Fujisaka S, Vienberg S, Softic S, et al. Interactions between Gut Microbiota, Host Genetics and Diet Modulate the Predisposition to Obesity and Metabolic Syndrome. Cell Metab. 2015;22:516-30.

4. Nurielohayon $\mathrm{M}$, Neuman $\mathrm{H}$, Koren $\mathrm{O}$. Microbial changes during pregnancy, birth, and infancy. Front Microbiol. 2016;7:1031.

5. Crusell MKW, Hansen TH, Nielsen T, Allin KH, Ruhlemann MC, Damm P, et al. Gestational diabetes is associated with change in the gut microbiota composition in third trimester of pregnancy and postpartum. Microbiome. 2018;6:89.

6. Ríoscovián D, Ruasmadiedo P, Margolles A, Gueimonde M, Reyesgavilán CGDL, Salazar N. Intestinal short chain fatty acids and their link with diet and human health. Front Microbiol. 2016;7:185.

7. Rahat-Rozenbloom S, Fernandes J, Gloor GB, Wolever TMS. Evidence for greater production of colonic short-chain fatty acids in overweight than lean humans. Int J Obes. 2014;38:1525-31.

8. Delzenne NM, Cani PD. Interaction between obesity and the gut microbiota: relevance in nutrition. Annu Rev Nutr. 2011;31:15-31.

9. Gao Z, Yin J, Zhang J, Ward RE, Martin RJ, Lefevre M, et al. Butyrate Improves Insulin Sensitivity and Increases Energy Expenditure in Mice. Diabetes. 2009;58:1509-17.

10. Perry RJ, Peng L, Barry NA, Cline GW, Zhang D, Cardone RL, et al. Acetate mediates a microbiomebrain-beta-cell axis to promote metabolic syndrome. Nature. 2016;534:213-7.

11. Zhu Q, Xie P, Li H, Ma C, Zhang W, Yin Y, et al. Fetal Huanjiang mini-pigs exhibit differences in nutrient composition according to body weight and gestational period. PloS One. 2018;13:e0199939.

12. Hu C, Yang Y, Li J, Wang H, Cheng C, Yang L, et al. Maternal Diet-Induced Obesity Compromises Oxidative Stress Status and Angiogenesis in the Porcine Placenta by Upregulating Nox2 Expression. Oxid Med Cell Longev. 2019; 2019: 2481592.

13. Kong X, Ji Y, Li H, Zhu Q, Blachier F, Geng M, et al. Colonic luminal microbiota and bacterial metabolite composition in pregnant Huanjiang mini-pigs: effects of food composition at different times of pregnancy. Sci Rep. 2017;6:37224.

14. Hu C, Li F, Duan Y, Yin Y, Kong X. Glutamic acid supplementation reduces body fat weight in finishing pigs when provided solely or in combination with arginine and it is associated with colonic propionate and butyrate concentrations. Food Funct. 2019;10:4693-704.

15. Ji Y, Kong X, Li H, Zhu Q, Guo Q, Yin Y. Effects of dietary nutrient levels on microbial community composition and diversity in the ileal contents of pregnant Huanjiang mini-pigs. PloS One. 2017;12:e0172086.

16. Amato KR, Yeoman CJ, Kent A, Righini N, Carbonero F, Estrada A, et al. Habitat degradation impacts black howler monkey (Alouatta pigra) gastrointestinal microbiomes. Isme J. 2013;7:1344-53. 
17. He Q, Ren $\mathrm{P}$, Kong $\mathrm{X}, \mathrm{Xu} \mathrm{W}$, Tang $\mathrm{H}$, Yin $\mathrm{Y}$, et al. Intrauterine growth restriction alters the metabonome of the serum and jejunum in piglets. Mol BioSyst. 2011;7:2147-55.

18. Koren O, Goodrich JK, Cullender TC, Spor A, Laitinen K, Bäckhed HK, et al. Host remodeling of the gut microbiome and metabolic changes during pregnancy. Cell. 2012;150:470-80.

19. Kennedy RC, Fling RR, Robeson MS, Saxton AM, Donnell RL, Darcy JL, et al. Temporal development of gut microbiota in triclocarban exposed pregnant and neonatal rats. Sci Rep. 2016;6:33430.

20. Le CE, Nielsen T, Qin J, Prifti E, Hildebrand F, Falony G, et al. Richness of human gut microbiome correlates with metabolic markers. Nature. 2013;500:541-6.

21. Dalby MJ, Ross AW, Walker AW, Morgan PJ. Dietary uncoupling of gut microbiota and energy harvesting from obesity and glucose tolerance in mice. Cell Rep. 2017;21:1521-33.

22. Huang $Y$, Zheng $Y$. The probiotic Lactobacillus acidophilus reduces cholesterol absorption through the down-regulation of Niemann-Pick C1-like 1 in Caco-2 cells. Bri J Nutr. 2010;103:473-8.

23. Linda A, Ying H, Paolo P, Marion KA, Janet $H$, Jan-Ake G, et al. Decreased Fat Storage byLactobacillus Paracaseils Associated with Increased Levels of Angiopoietin-Like 4 Protein (ANGPTL4). PloS One. 2010;5:855-67.

24. Wanchai K, Yasom S, Tunapong W, Chunchai T, Eaimworawuthikul S, Thiennimitr P, et al. Probiotic Lactobacillus paracasei HII01 protects rats against obese-insulin resistance-induced kidney injury and impaired renal organic anion transporter 3 function. Clin Sci. 2018;132:1545-63.

25. Baba E, Nagaishi S, Fukata T, Arakawa A. The role of intestinal microflora on the prevention of salmonella colonization in gnotobiotic chickens. Poult Scie. 1991;70:1902-7.

26. Barkeling B, Linne Y, Lindroos AK, Birkhed D, Rooth P, Rossner S. Intake of sweet foods and counts of cariogenic microorganisms in relation to body mass index and psychometric variables in women. Int J Obes Metab Disord. 2002;26:1239-44.

27. Ndanu TA, Aryeetey R, Sackeyfio J, Otoo G, Lartey A, Opintan JA, et al. Streptococcus mutans and lactobacillus species infection in obese and non-obese school children in Accra, Ghana. J Obes Overweight. 2015;1:1-5.

28. Zhao X, Guo Y, Liu H, Gao J, Nie W. Clostridium butyricum reduce lipogenesis through bacterial wall components and butyrate. Appl Microbiol Biotechnol. 2014;98:7549-57.

29. Eckburg PB, Bik EM, Bernstein CN, Purdom E, Dethlefsen L, Sargent M, et al. Diversity of the human intestinal microbial flora. Science. 2005;308:1635-8.

30. Abbeele PVD, Belzer C, Goossens M, Kleerebezem M, Vos WMD, Thas O, et al. Butyrate-producing clostridium cluster XIVa species specifically colonize mucins in an in vitro gut model. ISME J. 2013;7:949-61.

31. Hu C, Li F, Duan Y, Yin Y, Kong X. Dietary supplementation with leucine or in combination with arginine decreases body fat weight and alters gut microbiota composition in finishing pigs. Front Microbiol. 2019;10:1767.

32. Blaut M. Gut microbiota and energy balance: role in obesity. PNAS. 2015;74:227-34. 
33. Hong J, Jia Y, Pan S, Jia L, Li H, Han Z, et al. Butyrate alleviates high fat diet-induced obesity through activation of adiponectin-mediated pathway and stimulation of mitochondrial function in the skeletal muscle of mice. Oncotarget. 2016;7:56071-82.

34. McPherson RL, Ji F, Wu G, Blanton JR Jr, Kim SW. Growth and compositional changes of fetal tissues in pigs. J Anim Sci. 2004;82:2534-40.

\section{Tables}

Table 1. Concentrations of short-chain fatty acids in the intestine contents in different gestation stages (mg/g fresh contents).

\begin{tabular}{llccc}
\hline \multirow{2}{*}{ Items } & & \multicolumn{3}{c}{ Day of pregnancy } \\
\cline { 3 - 5 } & & 45 & 75 & 110 \\
\hline Jejunum & Acetate & $0.60 \pm 0.13^{\mathrm{a}}$ & $0.43 \pm 0.08^{\mathrm{b}}$ & $0.53 \pm 0.12^{\mathrm{ab}}$ \\
& Butyrate & $0.14 \pm 0.01$ & $0.17 \pm 0.04$ & $0.14 \pm 0.04$ \\
\hline Ileum & Acetate & $0.77 \pm 0.15^{\mathrm{b}}$ & $0.79 \pm 0.10^{\mathrm{b}}$ & $1.35 \pm 0.42^{\mathrm{a}}$ \\
& Butyrate & $0.21 \pm 0.06^{\mathrm{a}}$ & $0.10 \pm 0.01^{\mathrm{b}}$ & $0.14 \pm 0.02^{\mathrm{b}}$ \\
\hline Colon & Acetate & $3.25 \pm 0.85^{\mathrm{a}}$ & $3.01 \pm 0.85^{\mathrm{a}}$ & $1.58 \pm 0.41^{\mathrm{b}}$ \\
& Butyrate & $0.70 \pm 0.36$ & $0.60 \pm 0.20$ & $0.55 \pm 0.24$ \\
& Propionate & $1.36 \pm 0.33$ & $1.35 \pm 0.53$ & $0.96 \pm 0.59$ \\
& Valerate & $0.11 \pm 0.03$ & $0.13 \pm 0.03$ & $0.10 \pm 0.03$ \\
& Isobutyrate & $0.10 \pm 0.03$ & $0.10 \pm 0.02$ & $0.09 \pm 0.01$ \\
& Isovalerate & $0.10 \pm 0.06$ & $0.11 \pm 0.04$ & $0.09 \pm 0.02$ \\
\hline
\end{tabular}

Data represent as means $\pm \mathrm{SD}$; different letters within the same row indicate significant difference $(P<0.05)$.

Table 2. Concentrations of bioamines in the intestine contents in different gestation stages ( $\mu \mathrm{g} / \mathrm{g}$ fresh contents).

\begin{tabular}{llccc}
\hline Items & & \multicolumn{3}{c}{ Day of pregnancy } \\
\cline { 3 - 5 } & & 45 & 75 & 110 \\
\hline Jejunum & Cadaverine & $12.37 \pm 7.76$ & $14.91 \pm 8.26$ & $12.35 \pm 7.29$ \\
& Putrescine & $13.31 \pm 3.46^{\mathrm{a}}$ & $12.83 \pm 5.66^{\mathrm{a}}$ & $6.07 \pm 2.56^{\mathrm{b}}$ \\
& Spermidine & $4.25 \pm 1.57^{\mathrm{b}}$ & $4.15 \pm 0.66^{\mathrm{b}}$ & $6.19 \pm 1.66^{\mathrm{a}}$ \\
& Spermine & $4.83 \pm 1.62^{\mathrm{b}}$ & $4.36 \pm 2.51^{\mathrm{b}}$ & $8.80 \pm 4.06^{\mathrm{a}}$ \\
\hline Ileum & Cadaverine & $7.16 \pm 2.40$ & $6.72 \pm 1.41$ & $5.84 \pm 2.73$ \\
& Putrescine & $13.38 \pm 6.94^{\mathrm{a}}$ & $14.17 \pm 2.85^{\mathrm{a}}$ & $5.70 \pm 2.75^{\mathrm{b}}$ \\
& Spermidine & $4.03 \pm 1.76$ & $3.00 \pm 0.83$ & $3.07 \pm 1.38$ \\
& Spermine & $3.73 \pm 2.26$ & $2.64 \pm 1.63$ & $2.65 \pm 1.78$ \\
\hline Colon & Cadaverine & $9.09 \pm 3.09^{\mathrm{a}}$ & $5.61 \pm 2.71^{\mathrm{b}}$ & $3.55 \pm 1.99^{\mathrm{b}}$ \\
& Putrescine & $9.85 \pm 3.78$ & $7.88 \pm 3.58$ & $7.63 \pm 3.07$ \\
& Spermidine & $14.66 \pm 2.65$ & $17.10 \pm 4.97$ & $16.30 \pm 3.66$ \\
& Spermine & $0.94 \pm 0.22^{\mathrm{a}}$ & $1.02 \pm 0.31^{\mathrm{a}}$ & $0.46 \pm 0.15^{\mathrm{b}}$ \\
\hline
\end{tabular}

Data represent as means $\pm \mathrm{SD}$; different letters within the same row indicate significant difference $(P<0.05)$.

\section{Figures}


A

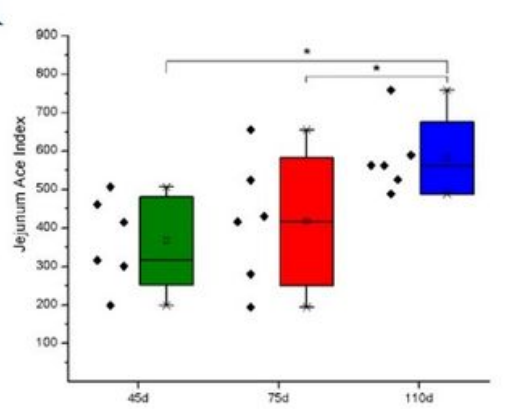

D

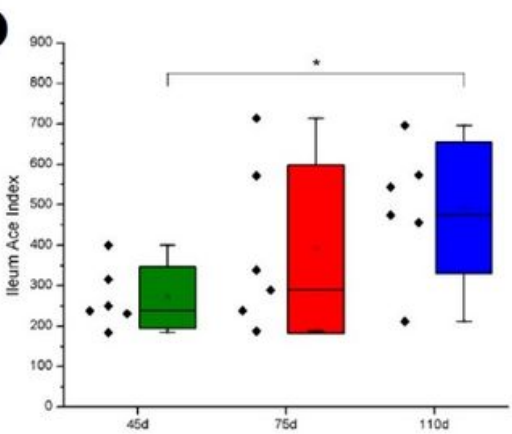

G
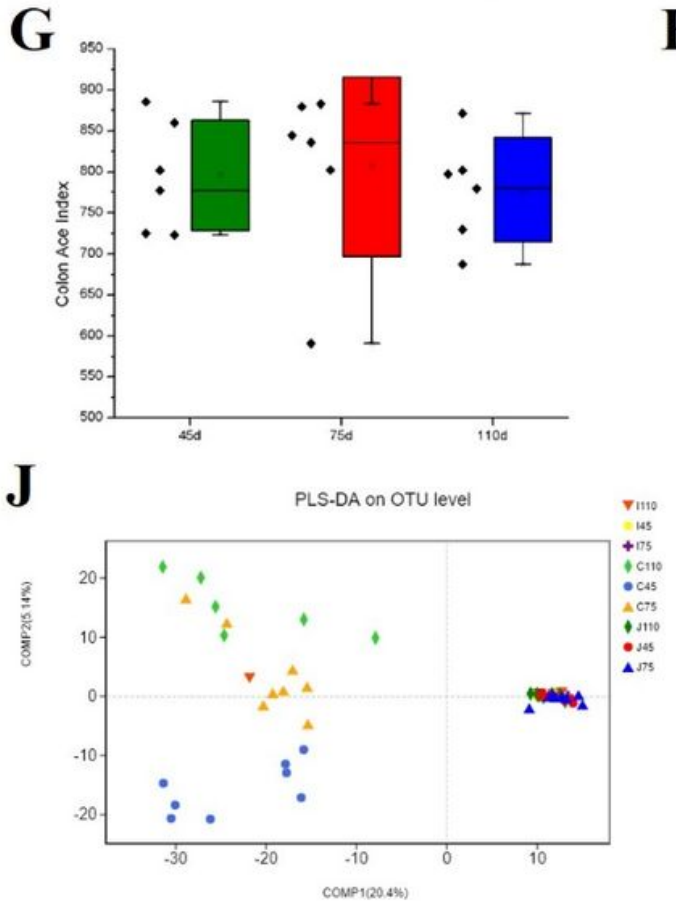

B

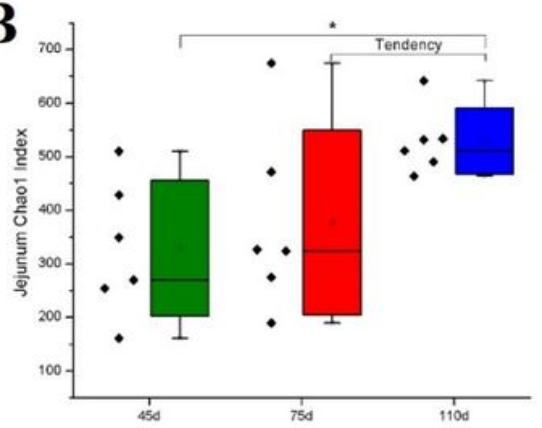

$\mathbf{E}$

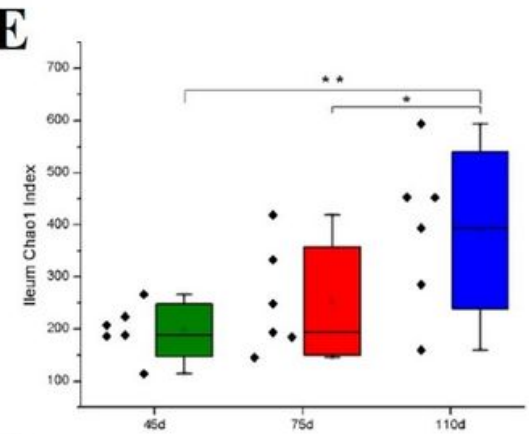

$\mathbf{H}$

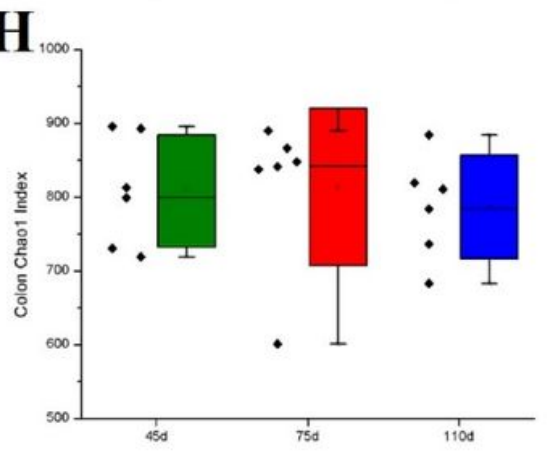

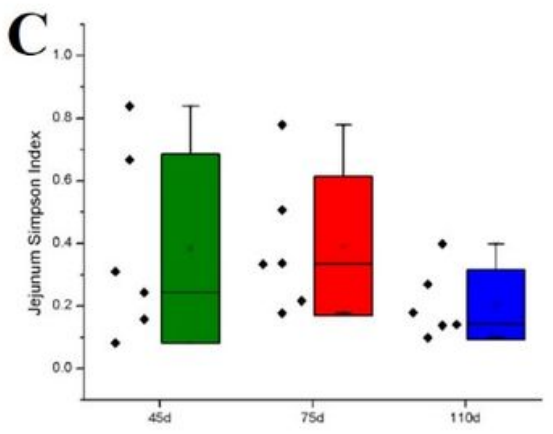

$\mathbf{F}$

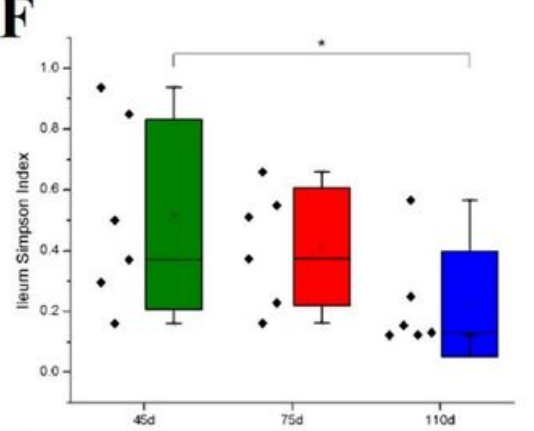

I

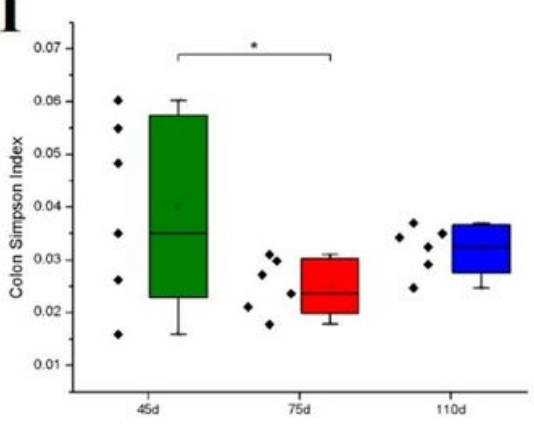

\section{Figure 1}

Alpha diversity indices of microbiota communities in jejunal, ileal, and colonic contents. (A-I) The microbiota diversity estimated by ACE, Chao1, and Simpson indices, respectively. (J) PLS-DA of microbiota community. $n=7-8 /$ group. 

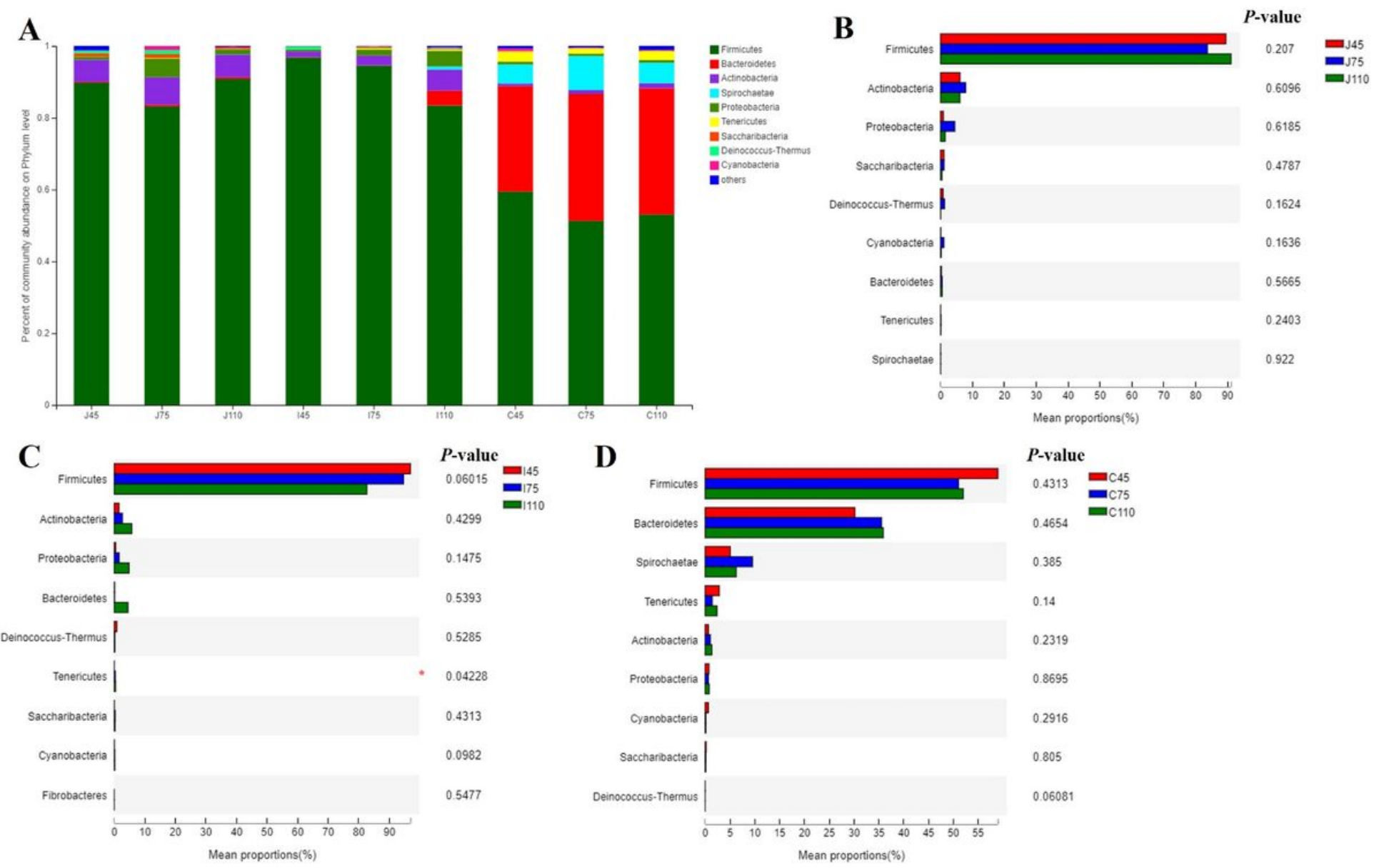

Figure 2

Microbiota communities at phylum level. (A) Distribution of microbiota at phylum level. Phylum with proportion $<0.01$ were grouped in others. Comparison of relative microbiota abundance in jejunal (B), ileal (C), and colonic (D) at phylum level. Differences were analyzed using Kruskal Wallis test; $* \mathrm{P}<0.05$; $n=7-8 /$ group. 

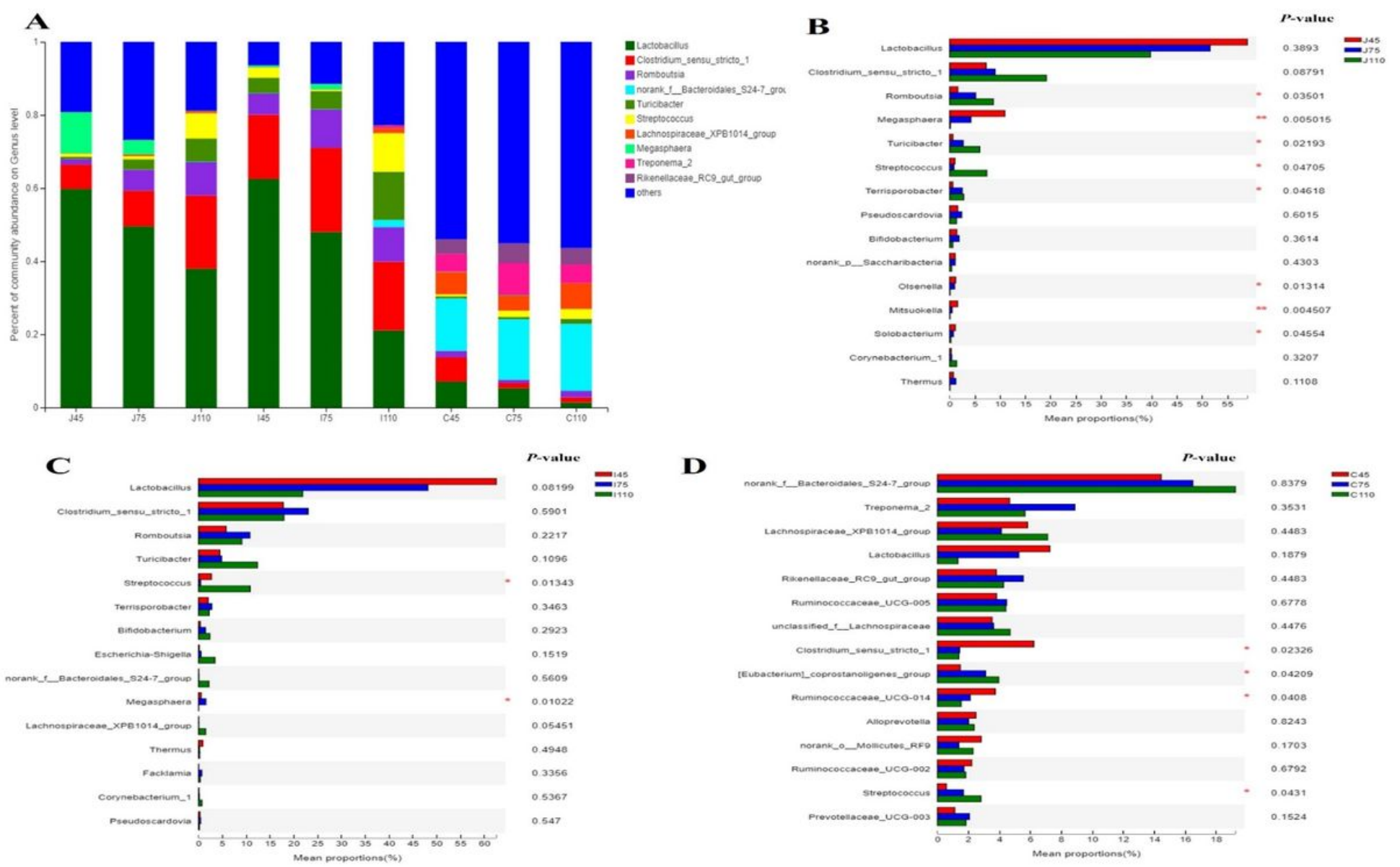

Figure 3

Microbiota communities at genus level. (A) Relative abundance of the 10 most abundant microbiota genera in different groups. Comparison of relative abundance in jejunal (B), ileal (C), and colonic (D) contents at genus level. Relative abundance of the 15 most abundant microbiota genera in different groups was listed. Differences were analyzed using Kruskal Wallis test; $* \mathrm{P}<0.05 ; \mathrm{n}=7-8 /$ group.

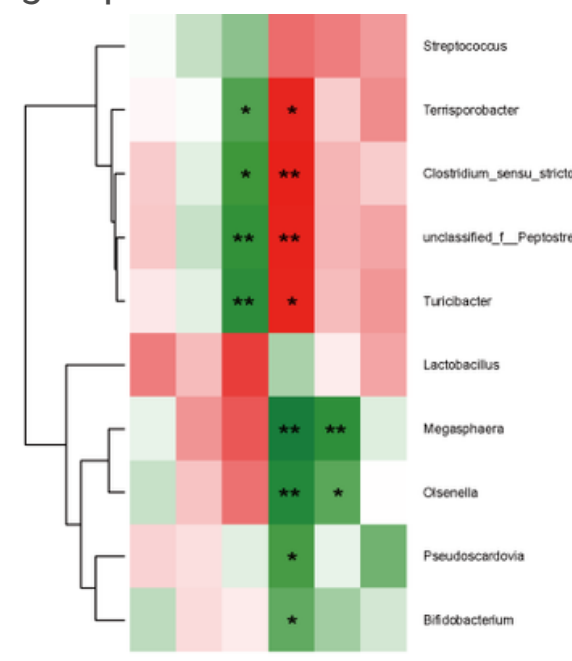

A 1715

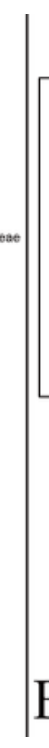

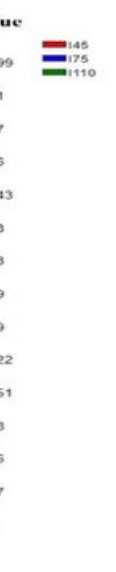


Pearson's linear correlation heatmap of microbiota metabolic and dominant genus microbiota in jejunal $(A)$, ileal $(B)$, and colonic $(C)$ contents. * in green grid indicates a negative correlation $(P<0.05)$ between the abundance of the genus microbiota and microbiota metabolites, whereas in the red grid indicates a positive correlation $(P<0.05)$
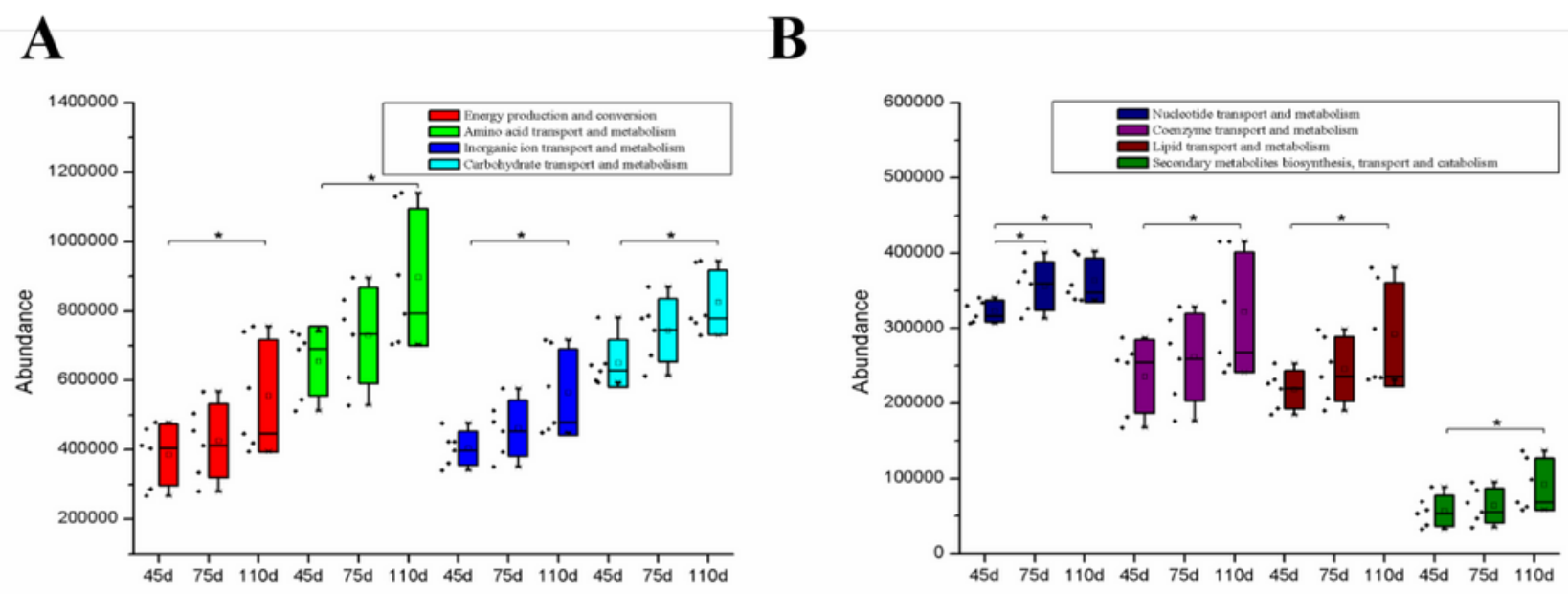

C

D
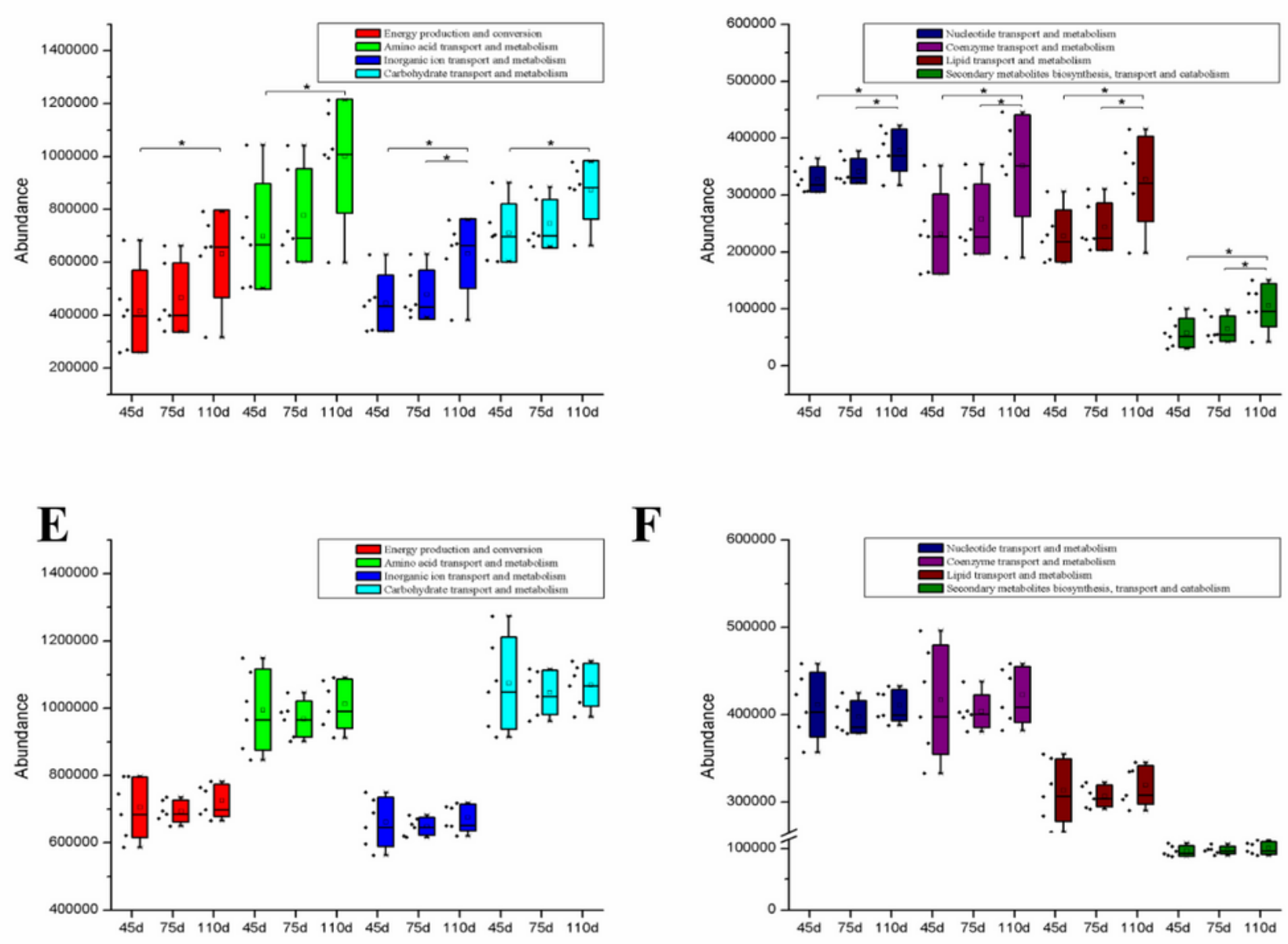

Figure 5 
Function prediction of the microbiota communities in jejunal ( $A$ and $B)$, ileal ( $C$ and $D)$, and colonic ( $E$ and F) contents.

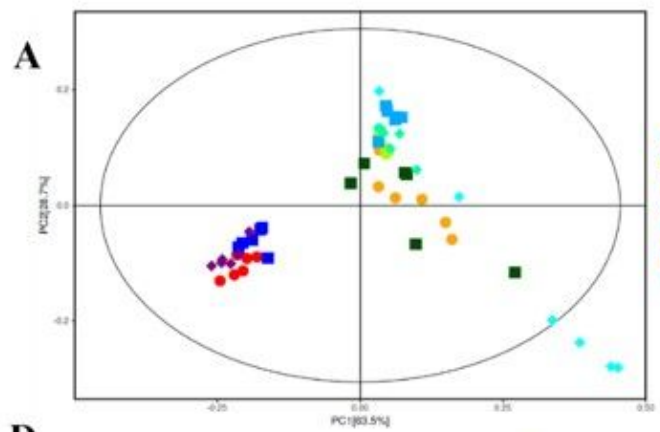

D

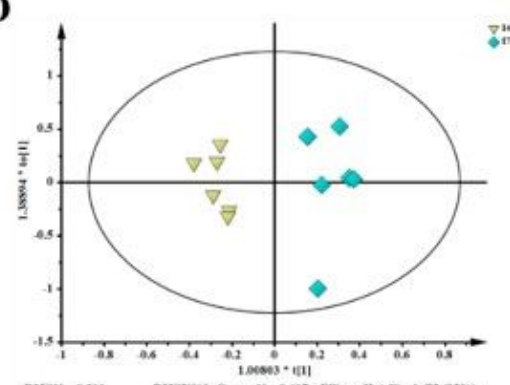

G

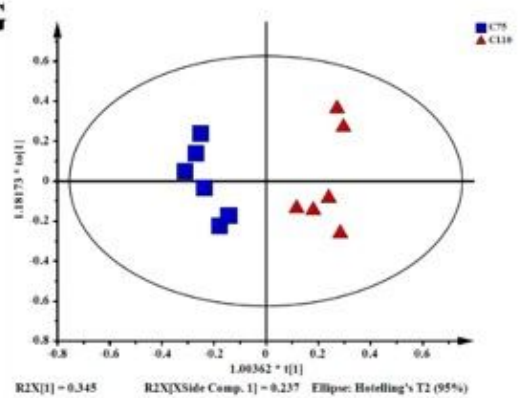

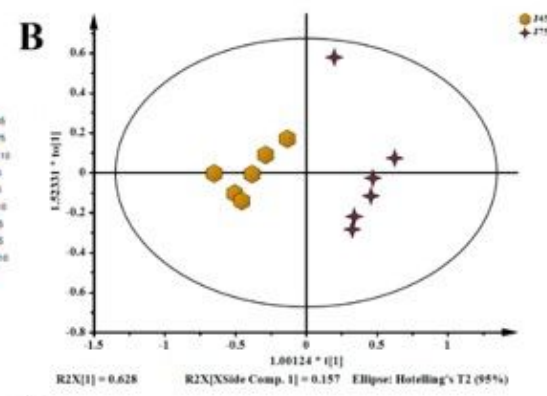

$\mathbf{E}$

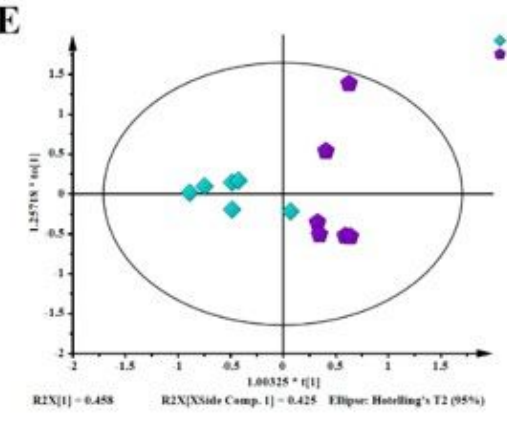

$+2$

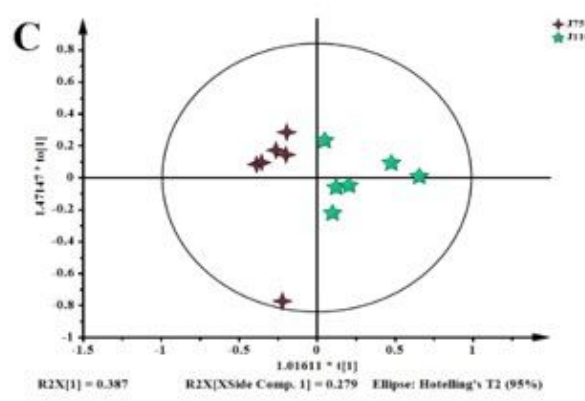

8

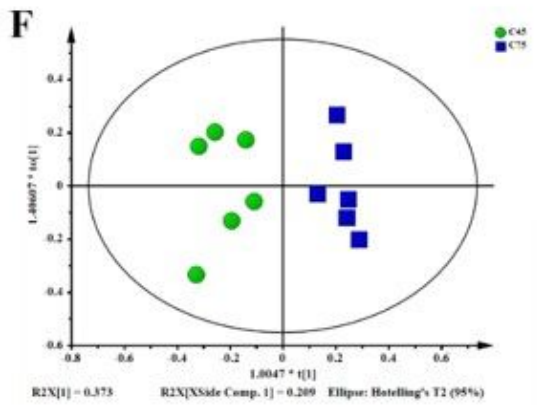

\section{Figure 6}

Cluster analysis of metabolome in intestinal contents. (A) Score scatter plot of PCA analysis for all samples. Score scatter plot of OPLS-DA analysis in jejunum (B and C), ileum (D and E), and colon (E and F).

A

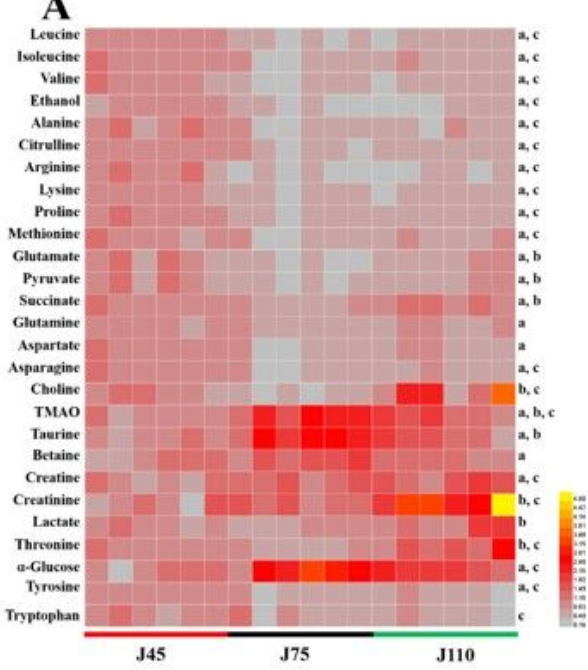

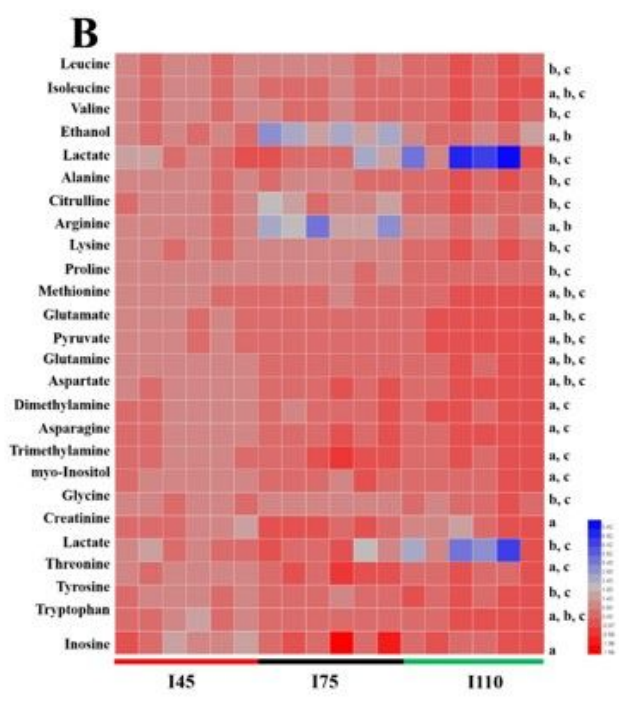

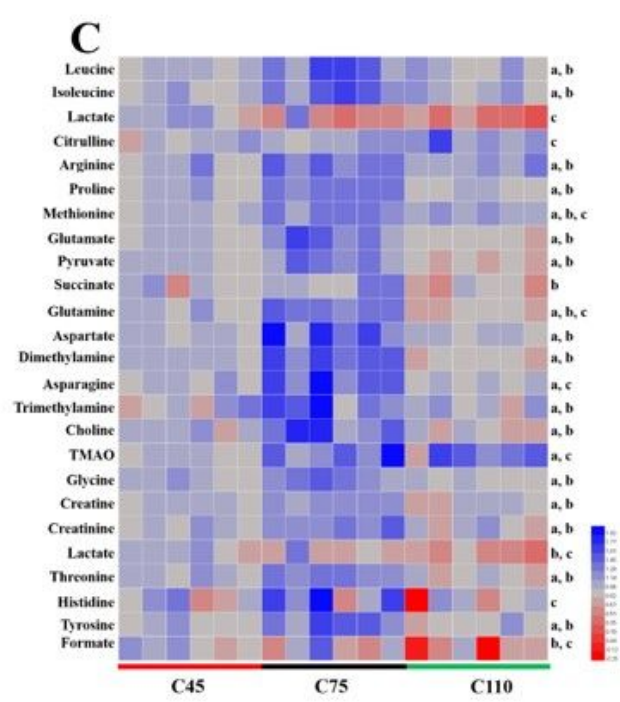


Figure 7

Heat-map of the metabolites in jejunal (D), ileal (E), and colonic (F) contents. The superscripts of $a, b$ and c indicated statistically significant difference $(P<0.05)$ between day 45 and day 75 , day 75 and day 110 , and day 45 and day 110 of gestation, respectively.

A

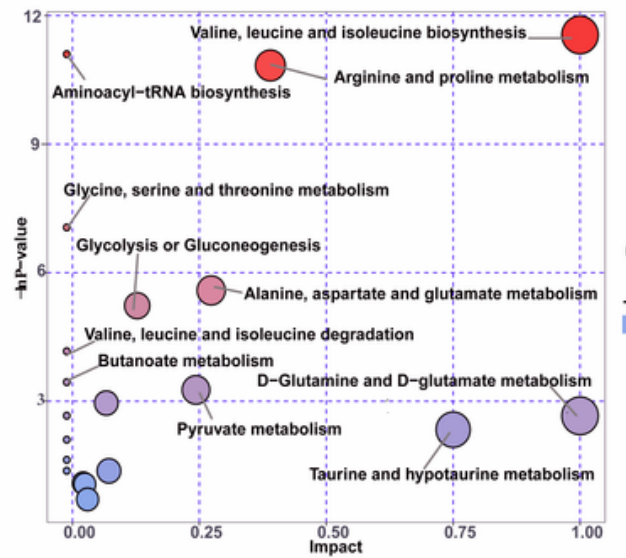

J45 VS J75

C

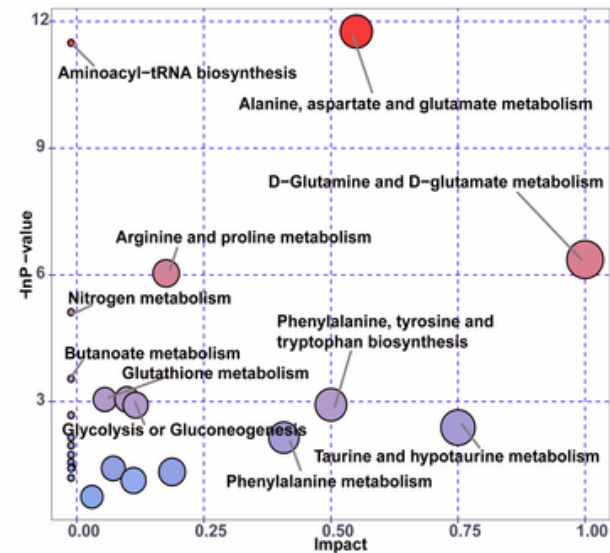

I45 VS I75

$\mathbf{E}$

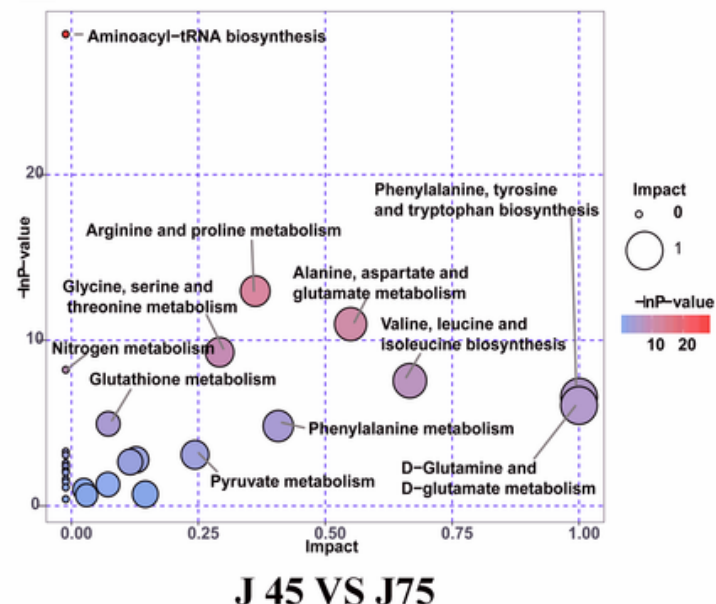

B

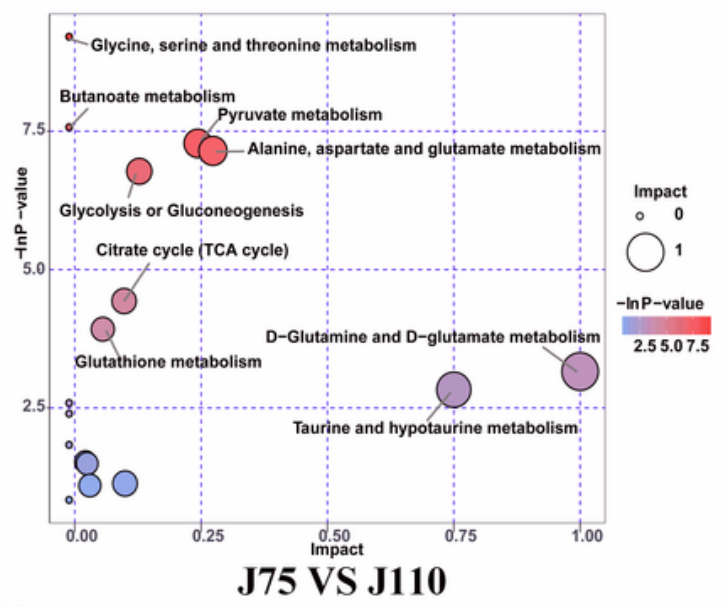

D

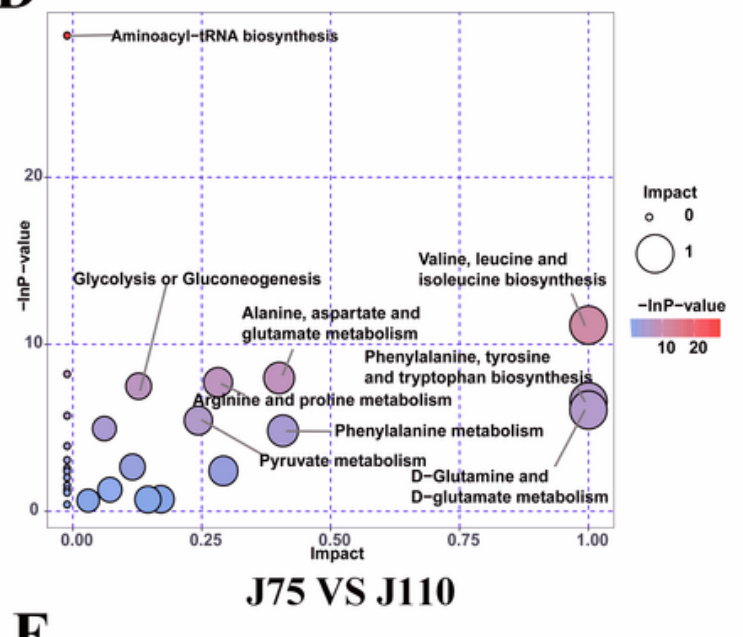

F

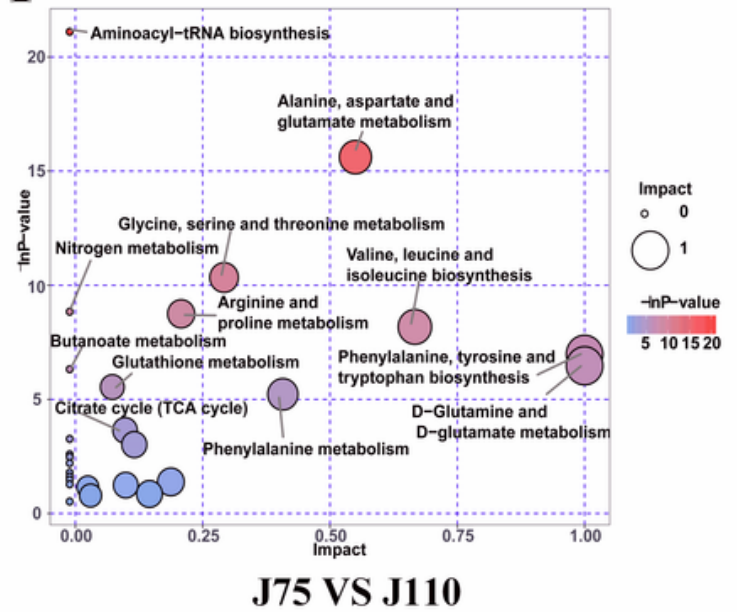

Figure 8 
Metabolic pathway enrichment analysis among jejunal ( $A$ and $B$ ), ileal ( $C$ and $D)$, and colonic ( $E$ and $F$ ) contents. The $x$-axis showed the pathway impact, and the $y$-axis provided the pathway enrichment. The size and color of the bubble indicate pathway enrichment and impact values.

\section{Supplementary Files}

This is a list of supplementary files associated with this preprint. Click to download.

- supplemental.docx 